\title{
Analogic Cellular PDE Machines
}

\author{
Csaba Rekeczky $^{+*}$, István Szatmári ${ }^{+}$, Péter Földesy ${ }^{+}$, and Tamás Roska ${ }^{+*}$ \\ ${ }^{+}$Analogical and Neural Computing Systems Laboratory \\ Computer and Automation Research Institute \\ Hungarian Academy of Sciences, Budapest, Hungary \\ *Jedlik Laboratories, Department of Information Technology \\ Péter Pázmány Catholic University, Budapest, Hungary
}

\begin{abstract}
This paper gives an overview on analogic cellular array architectures that can also be used to approximate partial differential equations (PDEs). Cellular arrays are massively parallel computing structures composed of cells placed on a regular grid. These cells interact locally and the array can have both local and global dynamics. The software of this architecture is an analogic algorithm that builds on analog and logical spatio-temporal instructions of the underlying hardware, that is a locally connected cellular nonlinear network (CNN, [1]-[5]). Within this framework two classes of PDEs, motivated also by image processing methodologies will be discussed: (i) reaction-diffusion (local) types and (ii) contrast modification (global) types. It will be shown that based on cellular diffusion and wave-computing formulations these classes can be approximated on existing CNN Universal Machine (CNN-UM, [4]) chips (e.g. [8]). Thus, the last generation of stored program topographic array microprocessors with integrated sensing and computing could also be viewed as the first prototypes of analogic cellular PDE MACHINES implemented on silicon.
\end{abstract}

\section{INTRODUCTION}

It is well known that all PDEs can be approximated to any desired accuracy by introducing finite differences [27] (and possibly discrete variables), i.e. they can always be mapped to a cellular structure. On the other hand, cellular arrays are computationally universal ([22]-[25]) and from an engineering point of view, they can be considered as dedicated architectures for building a universal parallel computer. The presentation of the next section aims to underline the engineering view that an efficient parallel PDE MACHINE, which can be built, must be a cellular array (e.g. [8]).

\section{PDE Machines Constructed as Cellular ARRAYS - AN ENGINEERING APPROACH}

In a continuous space-time approach diffusive and wavelike transport mechanisms in physics can be well characterized by partial differential equations (PDEs). A detailed qualitative and quantitative analysis of these processes usually focuses on space-time evolution of these systems; however, a closed form solution of the describing equations is available only in fairly simple cases. Then, one may consider on constructing a universal computer programmed by PDEs and capable of calculating all the solutions. The major obstacle in building the ideal PDE
$\mathrm{M}_{\mathrm{ACHINE}}$ - continuous in both space and time - stems from the fact that the "spectrum of the volume of any physical region is discrete" [20]. For instance, even when choosing some gas or fluid as a basic material, there is always a certain microscopic scale where these substances should be considered as spatially discrete. On the other hand, treating them on a macroscopic scale (thus continuous in space), another engineering problem arises: adjusting some of the (global) physical parameters of this system would mean a very restricted way of programming. As a consequence, it is very likely that this "computer" would be self-describing, capable of calculating and predicting only those processes that it actually represents.

Are there any better mathematical abstractions for parallel computing machines in engineering design?

Discretization along all spatial coordinates maps the PDE based system into a dynamical system described by ordinary differential equations (ODE). Stated with other words, the PDE is approximated on a fixed grid looking at its properties at a chosen scale (e.g. [29]-[32]). This leads to a cellular array structure in which each node represents an analog (continuous state and time) computing device. In this case, it is easier to imagine the corresponding universal computer composed of cells having a prescribed sphere of influence in a local neighborhood, similar to the sensory organs and the nervous system of different species in nature. Here, reprogramming would mean changing the cell types and/or their local interaction (in order to change the local configuration) to realize a universal computation.

Further simplification is possible by discretizing the ODE system in time and limiting the cell states to a finite number of states. This fully discrete system, described by ordinary difference-differential equations (ODDE), is theoretically still capable of reproducing the qualitative properties of the underlying PDE while possessing structural simplicity that makes it tailor-made for physical implementation. For instance, imagine a structure of molecules in a chemical substance where the spin of the electrons is described by discrete states and the chemical reaction evolves in phases. In this cellular array structure each node represents a digital (discrete state and time) computing device. Similarly to the analog case, reprogramming would ensure the construction of possible local configurations and the realization of a universal computation.

The above described analog/digital cellular arrays are not only good approximations of various PDE based dynamical 
systems, but they can also be considered as universal computers ([22]-[24]). Furthermore, it has been proven that there are cases in which for a certain cellular array structure (described by ODEs or local rules) the limiting PDE representation with the same qualitative properties does not even exist [21], [28]. This promotes a provocative view that PDEs are merely idealizations of (or alternatives to) cellular structures described by coupled ODEs or local update rules ([3], [25]).

As the generalized framework throughout the paper we have chosen a cellular array structure described by coupled nonlinear ODEs. The related $(\mathrm{CNN})$ formulation and various architecture prototypes will be introduced and discussed in the next section.

\section{CNN FORMULATION AND ARCHITECTURES}

Coupled nonlinear ODEs define a cellular neural/nonlinear network (CNN, [1]-[5]) structure composed of simple dynamical systems, called cells arranged at the node points of a regular grid. The state of any cells is represented by a real number, their interaction is local and the internal operation is continuous in time.

The cell dynamics is described by the following nonlinear ordinary differential equation with inter-cell coupling (the extension to higher dimensions is straightforward, allowing similar interlayer interactions):

$$
\begin{aligned}
C \frac{d}{d t} x_{i j}(t)=-R^{-1} x_{i j}(t) & +\sum_{k l \in N_{r}} A_{i j, k l} y_{k l}(t) \\
& +\sum_{k l \in N_{r}} B_{i j, k l} u_{k l}(t)+z_{i j} \\
y_{i j}(t)=f\left(x_{i j}(t)\right) &
\end{aligned}
$$

where $x_{i j}, u_{i j}, y_{i j}$ are the state, input and output voltage of the specified CNN cell, respectively. The notation $i j$ refers to a grid point associated with a cell on the $2 \mathrm{D} \mathrm{MxN}$ grid, and $k l \in N_{r}$ is a grid point in the neighborhood within the radius $r$ of the cell $i j . \mathrm{A}_{i j, k l}$ and $\mathrm{B}_{i j, k l}$ represent the linear feedback and the linear control, respectively. The constant $z_{i j}$ is the cell current, which could also be interpreted as a space-varying threshold. In general, the CNN template, which is the "program" of the CNN array, consist of the $\left[\begin{array}{lll}A & B & z\end{array}\right]$ terms (omitting the indices). The output characteristic $f$ is a sigmoid-type (e.g. piecewise-linear) function. The time constant of a (first order) $\mathrm{CNN}$ cell is determined by the linear capacitor $(\mathrm{C})$ and the linear resistor (R) and it can be expressed as $\tau=R C$. Without loss of generality $\mathrm{R}=1$ and $\mathrm{C}=1$ can be considered.

Remarks:

Equation (1) describes the standard CNN model ([1]-[3]). As it will be discussed later both the cell and the interaction types have been further generalized for numerous application areas.

Physical implementations with gray-scale I/O closely follow (1), except for some modifications limiting the dynamic range of the cell's state value ("full-range" model, [6],[8])

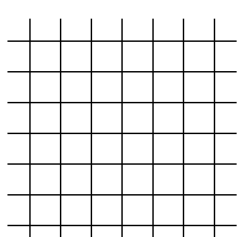

(a)

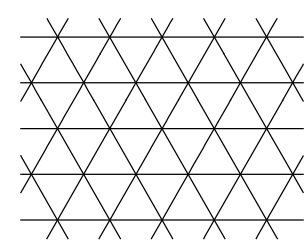

(b)

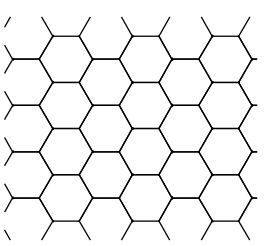

(c)

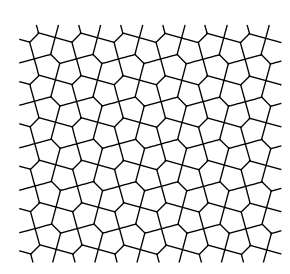

(d)

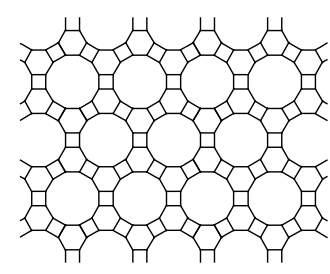

(e)
Figure 1 The most common and two special grid-types of cellular architectures. Regular contiguous tessellations of the plain based on congruent polygons are shown in the top row: (a) rectangular, (b), triangular and (c) hexagonal lattices. In the bottom row two special grid-types are given that belong to the ornamental group. All grid-types (b)-(e) could be mapped to a rectangular grid (a) with periodic space-variant interconnections [34].

The preceding definition of CNNs (1) can be further generalized and several types of specific cellular (nonlinear) arrays can be generated including a 2D cellular automata (CA, [26]).

They can be classified according to the types of the grid, the processor (cell), the interaction (template or update rule), and the mode of operation. Thus, building the core of a massively parallel computer based on cellular array architecture; the following key points should be addressed in detail:

\section{Grid types:}

Cellular arrays are usually defined on a spatially discrete square (rectangular) grid; however, hexagonal and triangular arrangements can also be considered (Fig. 1(a)(c)). These grids are the only regular contiguous tessellations of the plain based on congruent polygons alone. Other gridtypes can also be created based on non-regular congruent polygons or from a regular vertex grid through discrete geometrical transformations: rotations and translations (ornamental groups, Fig. 1(d)-(e)). A great number of these grids can be mapped on a typical eight-neighbor rectangular structure with periodic space-variant connections [34]. Multiple and varying grid sizes (e.g. course and fine grid; logarithmically changing size, etc.) may be useful in simulating adaptive biological systems (e.g. retina [33], LGN or magno/parvo pathways in the cortex).

\section{Cell (processor) types and boundary cell types:}

Cellular arrays can be built from linear/nonlinear, first or higher order cells. A linear or "small-signal" operation (as a special case) is achievable through a piece-wise linear output characteristic. A highly nonlinear dynamic behavior (typical CNN models) is attainable through a sigmoid; Gaussian or 
inverse-Gaussian type output characteristics. Cells can include additional local analog and/or logical memories for storing intermediate processing results. A cell might perform local logic operations (typical CA models) or a combined analogic (analog and logic) computation (extended CNN models). The cells of an array can be uniform or non-uniform (regularly or slightly varying). In some processing tasks two or three cell types in a regular grid might be very useful, for example, in color image processing.

Since in any physical implementation only a finite array can be built, a boundary condition should be exactly defined. Creating boundary cells that are not involved in the cooperative computation ("virtual cells") can satisfy this requirement. The most important boundary cell specifications are as follows: (i) Fixed (Dirichlet): constant values are assigned to all boundary cells; (ii) Zero-flux (Neumann): boundary cells are made to follow cells that are on the same side of the array; and (iii) Periodic (Toroidal): boundary cells are made to track the values of cells from the opposite side of the array.

\section{Neighborhood-size and interaction types:}

The interaction type (the biological "synapse") in between the grid cells represents the program of a cellular array. Depending on whether these interaction types are fixed or programmable, the array can be regarded as a specific purpose or a re-configurable parallel array architecture.

The nearest neighborhood is the most common sphere of influence in the inter-cell communication for both CA and CNN models (either the cross-shaped 4-connected or the starshaped 8 connected neighborhoods), however, larger neighborhood sizes can also be considered (typically in biological modeling or when creating adaptive artificial systems).

The interaction types can be linear or nonlinear memoryless functions (e.g. a typical CA update rule) of one, two or more variables. Delayed-nonlinear (e.g. a typical CNN template ) and dynamic (lumped) interactions are more general connection types. Breaking the symmetry or isotropy, furthermore, varying the nature of the interconnections in space and/or in time an extremely rich collection of further templates (or local update rules) can be created.

\section{Modes of the operation:}

The modes of operation can be continuous (general $\mathrm{CNN}$ models) or discrete time (general DTCNN and CA models). In the case of a discrete-time procedure the update mechanism can be synchronous or asynchronous. The computation can be analog, logic or analogic, and can be executed either in local mode or in propagating mode (on a de-coupled or coupled array, respectively). Besides the usual fixed-point operational mode (equilibrium computing), transient (non-equilibrium computing), oscillating, chaotic and general stochastic modes can also be exploited.

In the next two sections we will return to the basic formulation (1) and discuss the approximation of two important PDE classes.

\section{ApProximating Local PDEs}

Let us consider the following local PDE (a generalized reaction diffusion-type equation class [3]):

$$
\begin{aligned}
\frac{\partial \phi(x, y, t)}{\partial t}-\operatorname{div}(\operatorname{grad}(\phi(x, y, t))) & = \\
\mathfrak{I}_{1}\left(\phi_{0}\left(x, y, t_{0}\right)\right) & +\mathfrak{I}_{2}(\phi(x, y, t))
\end{aligned}
$$

where $\phi$ is the image intensity, $\phi_{0}$ represents the initial state and $\mathfrak{I}_{1}($.$) and \mathfrak{I}_{2}($.$) are nonlinear functions.$
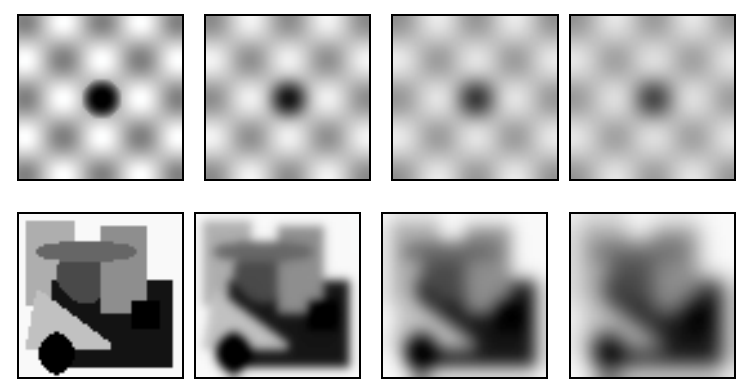

(a)
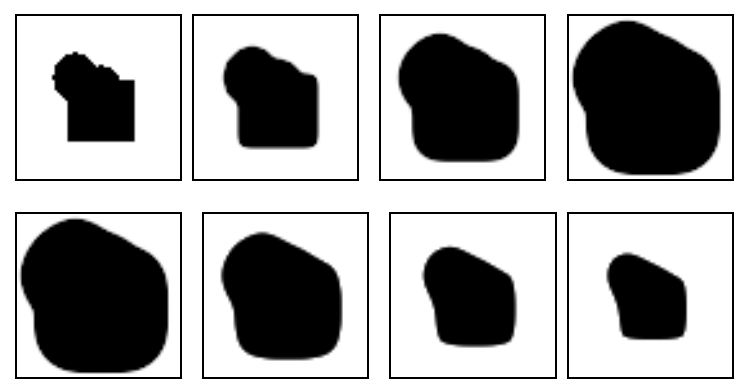

(b)

Figure 2 Programmable local PDE on a cellular nonlinear architecture: (a) controlled diffusion and (b) bi-directional trigger-wave propagation on a CNN-UM chip [8].

Assuming that the right hand side of (2) is zero we obtain the linear diffusion equation (at steady state the Laplace equation has to be solved). Dropping only the $\mathfrak{I}_{2}($.$) term$ results in a constrained linear diffusion equation $\left(\mathfrak{I}_{1}()\right.$. calculates a "spatial constrain"). If $\mathfrak{I}_{2}(.) \approx \operatorname{sigm}($.$) then a$ trigger-wave (or when $\mathfrak{I}_{1}() \neq$.0 a constrained trigger-wave) equation can be derived.

Using a finite difference approach all these equations naturally map to a cellular structure described by nonlinear ODEs (a CNN architecture). Let us consider an approximation that leads to existing architectures [8]:

$$
\begin{aligned}
& \frac{d \phi_{i j}(t)}{d t}=g\left(\phi_{i j}(t)\right)-c_{1} \phi_{i j}(t) \\
& +\frac{c_{1}}{4}\left(\phi_{i-1 j}(t)+\phi_{i+1 j}(t)+\phi_{i j-1}(t)+\phi_{i j+1}(t)\right)+z_{i j} \\
& \phi_{i j}(t)=f\left(x_{i j}(t)\right) \wedge g(.)=c_{0} f(.) \wedge z_{i j}=z_{0}+\sum_{k l \in N_{1}} b_{k l} \phi_{0, k l}
\end{aligned}
$$

where $\mathfrak{I}_{2}($.$) is replaced by g(),. f($.$) is a sigmoid-type$ function and $\mathfrak{I}_{1}($.$) is replaced by z_{i j}$ a linear combination of 
the input in the nearest neighborhood (plus an offset $z_{0}$ ). By adjusting the parameters $c_{0}, c_{1} \geq 0$ one can obtain different qualitative (e.g. linear diffusion or nonlinear trigger-wave) behaviors [11], [14], [15]. Experimental results can be found in Fig. 2.

While diffusion based filters can be primarily used in noise filtering, trigger-waves [15] provide a good approximation to some basic operations of differential morphology [19]. Several examples in Fig. 3 - Fig. 6. show how flat dilation, erosion and reconstruction can be implemented dynamically by these simple nonlinear waves (see also nonlinear wave metrics [13], [14]).

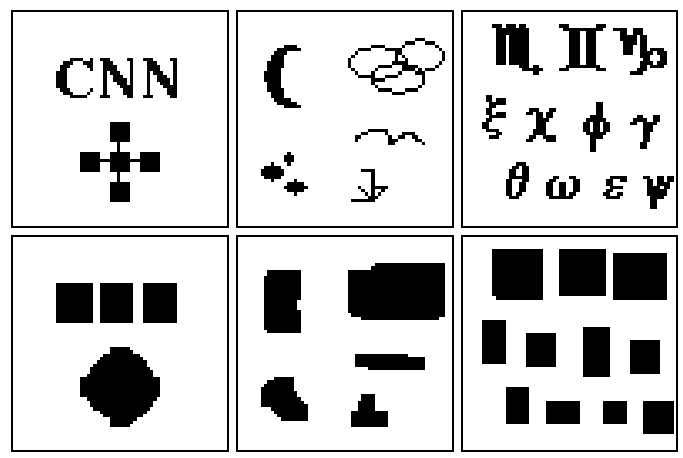

Figure 3 Trigger-wave generation from different initial conditions. Top row: simple objects given as initial conditions, bottom row: patches created by trigger-wave propagation.

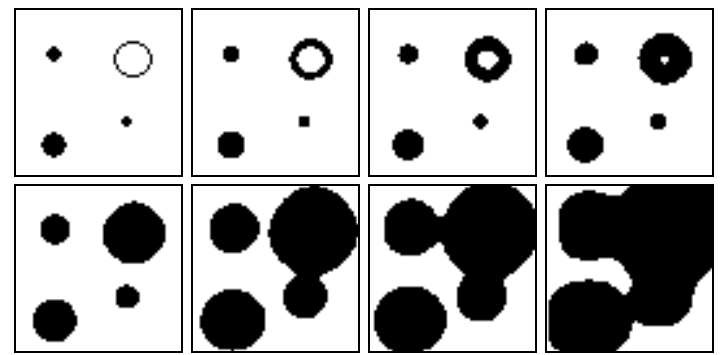

Figure 4 Trigger-wave generation from initial patches: a snapshot of the expanding wave front.

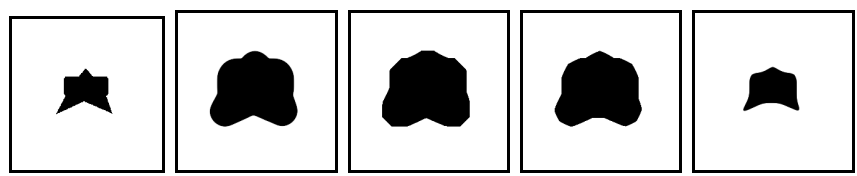

Figure 5 Trigger-waves generate multi-scale flat dilation and erosion operations of differential morphology.

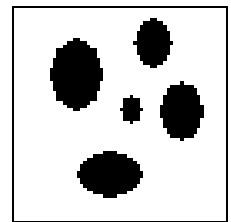

(a)

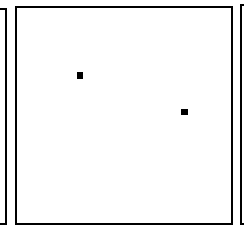

(b)

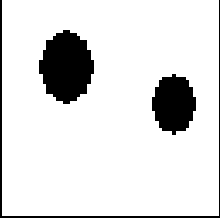

(c)
Figure 6 Recall/reconstruct operations implemented based on trigger-waves. (a) input image: a set of binary objects, (b) markers, (c) the output of the recall/reconstruct operation: labeled objects have been reconstructed.
Breaking the "synaptic" positivity, symmetry or isotropy in formulation (2) leads to patterns other than simple binary patches [9]-[10]. Some examples for the basic motifs and their combinations are illustrated in Fig. 7.

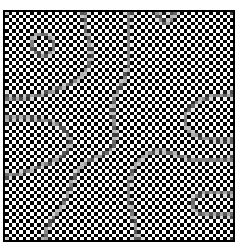

(a)

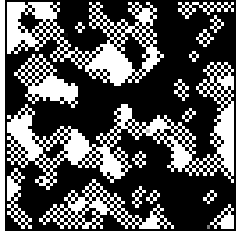

(d)

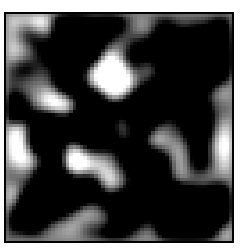

(b)

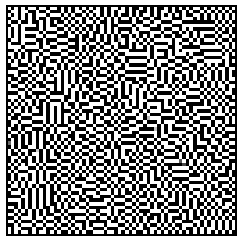

(e)

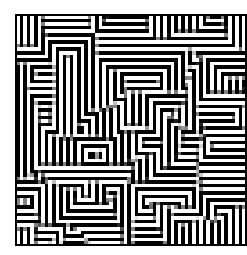

(c)
Figure 7 Programmable chemistry on a cellular architecture pattern creating self-organization phenomena: $(a)$ checkers, $(b)$ patches, and (c) stripes formed from random initial condition. A colorful combination of any two basic motifs can also be generated $(d)-(f)$.

\section{ApProximating GLOBAL PDEs}

Let us consider the following global PDE (a contrast modification-type equation class [16]-[18]):

$$
\begin{array}{r}
\frac{\partial \phi(x, y, t)}{\partial t}=\alpha \kappa+\left(N^{2}-H(\phi(x, y, t))\right) \\
-\mathrm{A}[(v, w): \phi(v, w, t) \geq \phi(x, y, t)]
\end{array}
$$

where $\phi$ is the image intensity, $\alpha$ and $N$ are constants, $\kappa$ is a regularizing term, $H($.$) represents a (strictly monotone)$ function and A[.] stands for area.

Mapping (4) into a nonlinear ODE let us consider the following two cases (both implementable as an analogic algorithm based on existing architectures [8]):

Case 1 (contrast enhancement through histogram equalization):

$$
\begin{aligned}
\alpha=0 ; H & =N^{2} / M ; N^{2}=1 ; M=1 ; \\
\frac{d \phi_{i j}(t)}{d t} & =-\phi_{i j}(t)+\left(1-A_{i j}\right)
\end{aligned}
$$

Case 2 (contrast enhancement through histogram modification and noise filtering):

$$
\begin{gathered}
\alpha=1 ; \quad \kappa=\operatorname{div}(\operatorname{grad}(\phi)) ; \\
H=N^{2} / M ; \quad N^{2}=1 ; \quad M=1 ; \\
\frac{d \phi_{i j}(t)}{d t}=-2 \phi_{i j}(t)+\left(1-A_{i j}\right)+ \\
\frac{1}{4}\left(\phi_{i-1 j}(t)+\phi_{i+1 j}(t)+\phi_{i j-1}(t)+\phi_{i j+1}(t)\right)
\end{gathered}
$$


In both cases $A_{i j}=$ const during the evolution, therefore should be calculated once for each cell. Since in any practical implementation only a finite number of gray-scale levels are considered (determined by the analog precision and/or the available digital representation) this also determines the number of distinct calculations necessary when approximating $A_{i j}$. Indeed, this can be done in parallel for all cells belonging to the same gray-scale level by using masked linear diffusion for normalized area estimation. Related implementation issues, algorithmic explanations, and detailed on-chip measurement results can be found in [12]. Some experimental result can be seen in Fig. 8.

Though $A_{i j}$ is the output of a global transformation it is possible to give an approximation based on purely local (analog \& logic) operations. Further improvement is also possible by embedding morphological processing at all distinct gray-scale levels. In the morphological "scalespace" one can efficiently combine histogram modification, smoothing and meaningful morphological image segmentation. This letter is often the main requirement of all subsequent processing blocks (see experimental results in Fig. 9 for an echocardiography image showing the 4chamber view of a human heart).
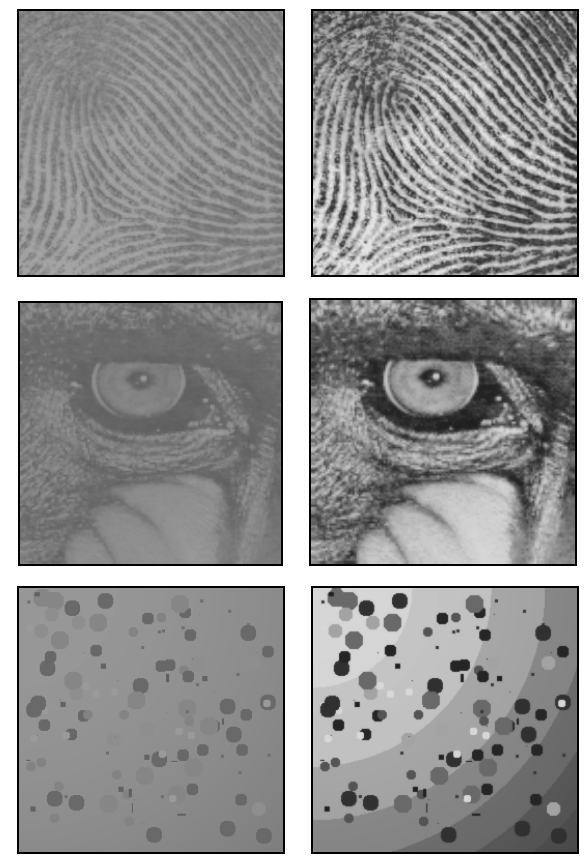

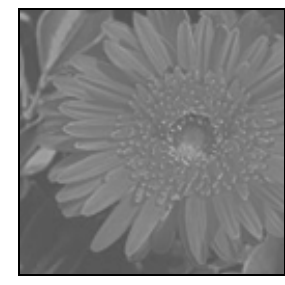

(a)

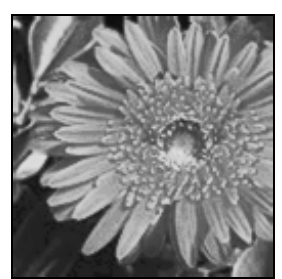

(b)
Figure 8 Programmable global PDE on a cellular nonlinear architecture - simultaneous contrast enhancement with noise suppression: (a) original, low contrast image, (b) enhanced, smoothed image.

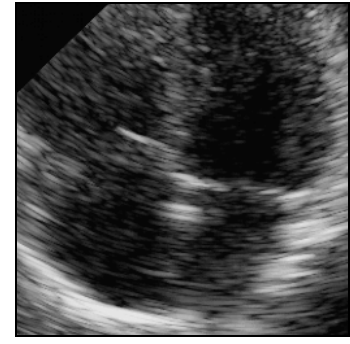

(a)

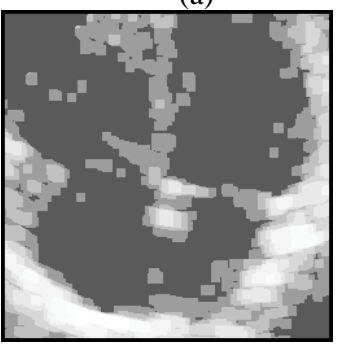

(c)

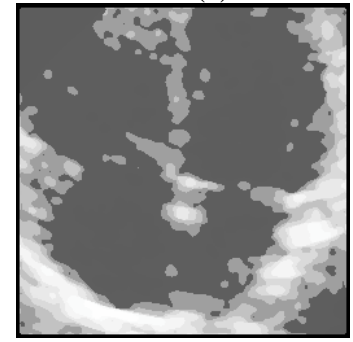

(e)

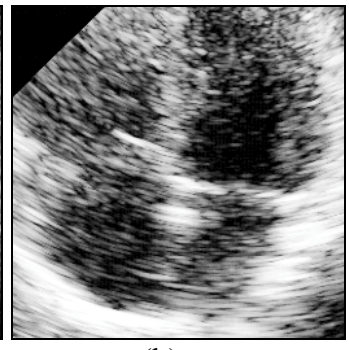

(b)

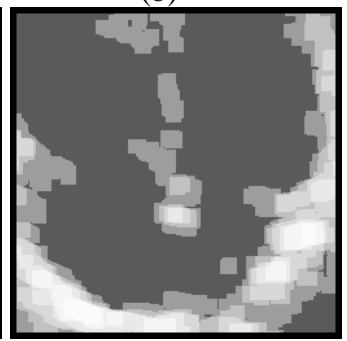

(d)

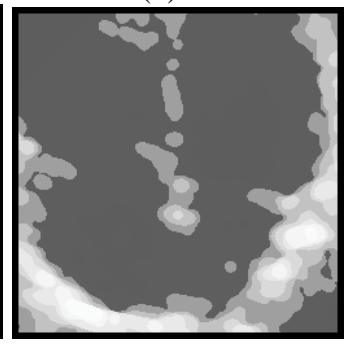

(f)
Figure 9 Programmable global PDE on a cellular nonlinear architecture - simultaneous contrast enhancement and noise filtering with embedded morphological processing of the levelsets: (a) original, low contrast image, (b) histogram equalized image, (c)-(d) histogram modification with embedded morphological processing (implemented as multi-step dilationerosion) at two different scales (3 steps and 5 steps) and 8 distinct gray-scale levels, (c)-(d) histogram modification with embedded differential morphological processing (implemented through expanding and shrinking trigger-waves) at two different scales $(3 \tau$ and $5 \tau)$ and 8 distinct gray-scale levels.

\section{Conclusions}

In this paper we have argued that efficient and realizable parallel PDE MACHINES, especially those that integrate sensing and computing should have a cellular nonlinear array architecture. It has been discussed and illustrated how certain important classes of local and global PDEs can be approximated by analogic cellular diffusion and wave-type algorithms. The focus has been put on formulations not exceeding the synaptic and algorithmic complexity that can be directly mapped and tested on available $\mathrm{CNN}-\mathrm{UM}$ chip prototypes (e.g. ACE4K [8]).

Acknowledgement: This work was financially supported by the Office of Naval Research (ONR) under the grant No. N68171-97-C9038. 


\section{REFERENCES}

[1] L. O. Chua and L. Yang, "Cellular Neural Networks: Theory and Applications", IEEE Trans. on Circ. \& Syst., Vol. 35, pp. 1257-1290, 1988.

[2] L. O. Chua, and T. Roska, "The CNN Paradigm", IEEE Trans. on Circ. \& Syst., Vol. 40, pp.147-156, 1993.

[3] L. O. Chua, "CNN: a Vision of Complexity", Int. J. of Bifurcation \& Chaos, Vol. 7, No. 10, pp. 2219-2425, 1997.

[4] T. Roska and L. O. Chua, "The CNN Universal Machine", IEEE Trans. on Circ. \& Syst., Vol. 40, pp. 163-173, 1993.

[5] P. P. Civalleri and M. Gilli, "On Stability of Cellular Neural Networks ", Journal of VLSI Signal Processing Systems, Vol. 23, pp. 429-436, Nov./Dec. 1999.

[6] S. Espejo, R. Carmona, R. Domingúez-Castro, and A. Rodrigúez-Vázquez, "CNN Universal Chip in CMOS Technology", Int. J. of Circuit Theory \& Appl., Vol. 24, pp. 93-111, 1996.

[7] Ari Paasio, Adam Dawidziuk, Kari Halonen and Veikko Porra, "Minimum Size 0.5 Micron CMOS Programmable 48 by 48 CNN Test Chip", in Proc. ECCTD '97, pp. 154-156, Budapest 1997.

[8] S. Espejo, R. Domínguez-Castro, G. Liñán, Á. RodríguezVázquez, "A $64 \times 64$ CNN Universal Chip with Analog and Digital I/O”, in Proc. ICECS'98, pp. 203-206, Lisbon 1998.

[9] K. R. Crounse, L. O. Chua, P. Thiran, and G. Setti, "Characterization and Dynamics of Pattern Formation in Cellular Neural Networks", IEEE Trans. on Circuits and Systems, Vol. 6, pp. 1703-1724, 1996.

[10] P. Thiran, K. R. Crounse, L. O. Chua, and M. Hasler, "Pattern Formation Properties of Autonomous Cellular Neural Networks", IEEE Trans. on Circuits and Systems, Vol. 42, pp. 757-774, 1995.

[11] Cs. Rekeczky, Á. Tahy, Z. Végh, and T. Roska, "Spatiotemporal Nonlinear Filtering and Endocardial Boundary Detection in Echocardiography", Int. J. of Circ. Theory \& Applic., Vol. 27, pp. 171-207, 1999.

[12] Cs. Rekeczky, Gy. Cserey, P. Földesy, and T. Roska, "PDE Based Histogram Modification with Embedded Morphological Processing of the Level-sets", to be presented at CNNA 2002, Frankfurt, 2002.

[13] V. Krinsky, V. Biktashev, and N. Efimov: "Autowaves principles for parallel image processing”, Physica D, Vol. 49, pp. 247-253, 1991.

[14] I. Szatmári, Cs. Rekeczky, T. Roska: "A Nonlinear Wave Metric and its CNN Implementation for Object Classification", Journal of VLSI Signal Processing, Vol.23. No.2/3. pp.437-448, Kluwer, 1999.

[15] Cs. Rekeczky and L. O. Chua: "Computing with Front Propagation: Active Contour and Skeleton Models in Continuous-Time CNN", Journal of VLSI Signal Processing, Vol.23. No.2/3. pp.373-402, Kluwer, 1999.

[16] G. Sapiro and V. Caselles, "Histogram Modifications via Differential Equations", Journal of Differential Equations, Vol. 135, pp. 238-268, 1997.

[17] G. Sapiro and V. Caselles, "Contrast Enhancement via Image Evolution Flows", Graphical Models and Image Processing, Vol. 59, pp. 407-416, 1997.
[18] V. Caselles, J-L. Lisani, and G. Sapiro, "Shape Preserving Local Histogram Modification", IEEE Trans. on Image Processing, Vol. 8, pp. 220-230, 1999.

[19] P. Maragos, "Differential Morphology and Image Processing", IEEE Trans. on Image Processing, Vol. 5, No. 6, 1996.

[20] C. Rovelli and L. Smolin, "Discreteness of Area and Volume in Quantum Gravity", Nucl. Phys. B, Vol. B442, pp. 593619, 1995.

[21] J. P. Keener, "Propagation and its Failure in Coupled Systems of Discrete Excitable Cells", SIAM J. Appl. Math., Vol. 47, 556-572, 1987.

[22] E. R. Berlekamp, J. H. Conway, and H. K. Guy, Winning Ways for your Mathematical Plays, Academic Press, New York, 1982.

[23] T. Toffoli, "Computation and Construction Universality of Reversible Cellular Automata", J. Comput Systems Sci., Vol. 15, p. 213, 1977.

[24] L. O. Chua, T. Roska, and P. L. Venetianer, "The CNN is as Universal as the Turing Machine", IEEE Trans. on Circuits and Systems, Vol. 40, pp. 289-291, April 1993.

[25] T. Toffoli, "Cellular Automata as an Alternative to (Rather than an Approximation of) Differential Equations in Modeling Physics", Physica 10D, pp. 117-127, 1984.

[26] N. H. Packard and S. Wolfram, "Two-dimensional Cellular Automata”, J. of Statistical Physics, Vol. 38, pp. 126-171, 1985.

[27] L. V. Kantorovich and V. I. Krylov, Approximate Methods of Higher Analysis, Interscience Publishers, New York, 1964.

[28] C. Serpico, G. Setti, P. Thiran, and A. Pascarelli, "Local Diffusion, Global Propagation and Propagation Failure in 1D Cellular Neural Networks", in Proc. NOLTA'99, pp. 399402, Hawaii 1999.

[29] T. Roska, D. Wolf, T. Kozek, R. Tetzlaff, and L. O. Chua, "Solving Partial Differential Equations by CNN", in Proceedings of European Conference on Circuit Theory and Design ECCTD '93, pp. 1477-1482, Davos, 1993.

[30] T. Roska, L. O. Chua, D. Wolf, T. Kozek, R. Tetzlaff, and F. Puffer, "Simulating Nonlinear Waves and Partial Differential Equations via CNN - Part I: Basic Techniques", IEEE Trans. on Circuits and Systems, Vol. 42, No. 10, pp. 807-815, October 1995.

[31] T. Kozek, L. O. Chua, T. Roska, D. Wolf, R. Tetzlaff, F. Puffer, K. Lotz, "Simulating Nonlinear Waves and Partial Differential Equations via CNN - Part II: Typical Examples", IEEE Trans. on Circuits and Systems, Vol. 42, No. 10, pp. 816-821, October 1995.

[32] T. Kozek and T. Roska, "A double time-scale CNN for solving 2-D Navier-Stokes equations", International Journal of Circuit Theory and Applications, Vol. 24, pp. 49-56, 1996.

[33] F. Werblin, T. Roska, and L. O. Chua, "The Analogic CNN Universal Machine as a Bionic Eye", International Journal of Circuit Theory and Applications, Vol. 23, pp. 541-569, 1995.

[34] A. Radványi, "On the Rectangular Grid Representation of General CNNs", in Proc of the 6th IEEE International Workshop on Cellular Neural Networks and their Applications CNNA 2000, pp. 387-394, Catania, May 2000. 